Meta

Journal des traducteurs

Translators' Journal

\title{
Terminologie, service compris
}

\section{Noëlle Guilloton}

Volume 36, numéro 1, mars 1991

La terminologie dans le monde : orientations et recherches

URI : https://id.erudit.org/iderudit/002987ar

DOI : https://doi.org/10.7202/002987ar

Aller au sommaire du numéro

Éditeur(s)

Les Presses de l'Université de Montréal

ISSN

0026-0452 (imprimé)

Découvrir la revue

Citer cet article

Guilloton, N. (1991). Terminologie, service compris. Meta, 36(1), 214-217.

https://doi.org/10.7202/002987ar

Ce document est protégé par la loi sur le droit d'auteur. L'utilisation des services d'Érudit (y compris la reproduction) est assujettie à sa politique d'utilisation que vous pouvez consulter en ligne.

https://apropos.erudit.org/fr/usagers/politique-dutilisation/
Cet article est diffusé et préservé par Érudit.

Érudit est un consortium interuniversitaire sans but lucratif composé de l’Université de Montréal, l'Université Laval et l'Université du Québec à Montréal. Il a pour mission la promotion et la valorisation de la recherche. https://www.erudit.org/fr/ 


\section{TERMINOLOGIE, SERVICE COMPRIS}

NOËLLE GUILLOTON

Office de la langue française, Montréal, Canada

La recherche terminologique ponctuelle est connue et pratiquée dans la plupart des milieux où l'on dispense des services linguistiques. Sa méthodologie a été établie voici plusieurs années; elle est maintenant enseignée comme partie intégrante des programmes de cours de terminologie. Définie comme «une technique d'investigation d'un terme ou d'un groupe restreint de termes appartenant à n'importe quel domaine de l'activité humaine, en vue de satisfaire un besoin immédiat exprimé par un usager», la recherche terminologique ponctuelle doit allier d'une part rigueur et précision, et d'autre part adéquation au contexte de la demande et efficacité de la communication. Voilà des impératifs qui ne sont guère faciles à concilier, comme l'expérience nous le fait voir quotidiennement.

Nous ne reprendrons ici ni les différentes étapes de la méthodologie, ni la typologie des cas, toujours valable; nous nous attacherons plutôt à situer la recherche terminologique ponctuelle dans le cadre d'une activité globale de consultation au sein d'un service d'assistance linguistique qui possède des dimensions et des prolongements extra-terminologiques, avec ses exigences et ses limites.

L'activité de consultation trouve sa raison d'être dans les besoins nombreux et pressants que manifestent sans cesse ceux et celles qui, en situation de communication écrite ou orale, désirent transmettre un message clair, précis, efficace, dont la qualité est conforme aux attentes de ceux et celles à qui il est destiné. Besoins d'être informé, guidé, ou rassuré, exprimés par des personnes conscientes de l'importance sociale du code qu'est la langue et ressentant sans doute une certaine insécurité linguistique. Il s'agit donc pour elles d'obtenir l'avis d'experts aptes à les comprendre et à les conseiller. La consultation est gratuite et n'est pas assortie de l'obligation de la suivre (il y a des conseilleurs, mais pas de payeurs!), bien que dans le cas d'un service public comme à l'Office de la langue française, les usagers soient des contribuables qui, en tant que tels, font explicitement valoir leurs droits aux services d'assistance linguistique autant qu'aux autres services offerts par l'Administration. Une grande part de son efficacité repose donc sur la bonne foi et la bonne volonté du demandeur.

Mais qui est ce demandeur?

On ne peut le définir au singulier tant il serait illusoire d'essayer de dégager un profil de l'usager type. Le public de l'assistance linguistique et terminologique est pluraliste. Composé surtout de personnes au travail, souvent dans un contexte de francisation, il comprend langagiers, communicateurs, professeurs, publicitaires, écrivants dans tous types de situations, personnel de secrétariat, commerçants, imprimeurs, juristes... À l'heure du clientélisme et de l'insistance sur la qualité du service aussi bien dans l'Administration que dans le secteur privé, - ce sera le leitmotiv des années 90 - ce public est assidu et exigeant. En quoi consistent donc ces services qu'il attend et quels rapports ceux-ci ont-ils avec la recherche terminologique ponctuelle?

La pratique de l'assistance terminologique et linguistique est multiforme, complexe. Si la typologie des cas exposée dans la Méthodologie de la recherche 
terminologique ponctuelle est exacte, il ne faut pas la confondre avec une typologie des situations de consultation, qui sont beaucoup plus diverses et font appel à une panoplie de ressources matérielles et documentaires d'une part, aussi bien qu'intellectuelles et humaines d'autre part. Il s'agit ici d'un service personnalisé, de conseils sur mesure et non pas de renseignements à la chaìne, même si le rythme de travail et la facilité avec laquelle on quantifie cette activité pourrait laisser entendre le contraire.

Les personnes qui s'adressent à la Division des consultations de l'Office de la langue française (nous les avons brièvement présentées plus haut) le font pour des raisons qu'elles jugent importantes et souvent après avoir épuisé les ressources - plus ou moins vastes - à leur disposition. Il peut s'agir parfois d'une simple curiosité mais, étant donné le sérieux qu'on accorde aux questions de langue au Québec et la relative difficulté d'accès du service, c'est plutôt le souci de la qualité de la langue qui les guide et, au delà d'une réponse à leur interrogation, la volonté d'en référer à l'autorité suprême en matière de langue, le désir de connaître et de respecter la norme, de ne pas faire de faute, de ne pas être critiqué. La ou le terminologue est ainsi appelé à arbitrer, à trancher entre des parties, à expliquer, voire à interpréter phrases ou expressions, à diffuser la terminologie et les prises de position officielles ou même à exposer les incertitudes de l'usage en étant bien conscient que, pour le public, c'est toujours l'Office de la langue française qui parle par sa bouche... et que c'est l'image de l'Office qu'il contribue à construire.

Quelles sont alors les ressources sur lesquelles il faut pouvoir compter pour une assistance terminologique et linguistique efficace?

Ressources intellectuelles et humaines d'abord. Elles sont évidemment capitales. Les terminologues généralistes qui assurent ce service doivent posséder les qualités et compétences suivantes: maîtrise de la méthodologie, intégration de connaissances linguistiques à jour, culture générale, curiosité et souplesse intellectuelles, intuition et vivacité d'esprit, capacité de réagir dans des délais très courts, autonomie aussi bien qu'aptitude à travailler en équipe, jugement sûr, bon sens, esprit de synthèse, vision de ce qui est nécessaire et suffisant (ce qui permet de mettre «un terme» à certaines recherches), respect de l'interlocuteur, pédagogie et persuasion, esprit de service... Voilà quels doivent être les atouts des terminologues affectés à cette tâche aussi astreignante qu'intéressante.

Ressources personnelles nous l'avons vu, ressources matérielles aussi évidemment.

Qui dit terminologie dit banques de terminologie. L'assistance terminologique à une grande échelle ne peut plus se concevoir sans elles, même si celle-là ne se borne pas, tant s'en faut, à l'interrogation de celles-ci. Les banques de terminologie sont particulièrement utiles en recherche bilingue, ou recherche de l'équivalent, en recherche de l'attestation du terme et en recherche du sens du terme. Pour la recherche du terme correspondant à la notion, opération d'encodage, démarche essentiellemnt terminologique en fait, les fichiers automatisés sont d'une utilité moindre, pour ne pas dire nulle. Il faut procéder par tâtonnement ou s'en servir pour contrevérifier les résultats obtenus par cl'autres moyens.

Une assistance terminologique et linguistique touchant à tous les domaines de l'activité humaine exige également une documentation encyclopédique, disponible et à jour. Ouvrages lexicographiques et autres, périodiques (même si leur exploitation et leur dépouillement exigent un temps dont on ne dispose pas souvent), accès aux banques de données documentaires, en plus des sources orales que constituent les spécialistes mis à contribution le cas échéant.

Comptant sur ces ressources documentaires et à même de les exploiter, les terminologues bien formés font face à des demandes où terminologie, grammaire et typographie sont étroitement liées. L'usager qui veut connaître l'équivalent français de 
fax pose non seulement une question de terminologie, mais aussi une question de typographie où le contexte et le support en cause sont très importants, voire déterminants: s'agit-il d'une inscription sur une carte professionnelle? D'une désignation sur une facture? D'un élément d'une future raison sociale? L'usagère qui veut simplement vérifier le féminin d'une appellation d'emploi a aussi besoin qu'on s'assure de la correction de l'appellation elle-même (... elle ne sera jamais «superintendante»!) et qu'on la conseille sur la façon d'insérer les féminins dans les textes. Et comment dire si fibre(s) alimentaire(s) s'emploie nécessairement au pluriel, à moins de faire une recherche dans la documentation spécialisée? La personne qui rencontre le mot sousministériat veut savoir ce qu'il désigne, où et quand elle peut employer ce statisme. Entre cassonade et sucre roux, que choisir? L'Énoncé d'une politique linguistique relative aux québécismes est un bon guide, tout comme celui sur l'emprunt des formes linguistiques étrangères, qui justifie le fait d'écarter feedback et de conserver hot-dog. Les terminologues sont ici des vulgarisateurs, elles et ils expliquent les raisons de tel ou tel choix que devrait faire l'usager. Lorsqu'ils répondent à une question de grammaire ou d'orthotypographie (ah! ces majuscules), ils conseillent aussi l'ouvrage qui a fourni la réponse, expliquent comment le consulter, conscients qu'ils sont de la dimension didactique de leur rôle: il ne s'agit pas là de fidéliser la clientèle, mais bien au contraire de rendre le public aussi autonome que possible dans la production de textes de qualité.

Cet objectif d'autonomisation est dicté par le réalisme qu'il faut garder lorsqu'on assure une telle assistance, qui pourrait se comparer au tonneau des Danaïdes. D'une part, les besoins sont immenses et les ressources quantitativement limitées (et dans quelle mesure un accès facilité à des services accrus n'encouragerait-il pas la dépendance, rendant superflu pour le public le recours à toute documentation?), d'autre part il faut pouvoir déterminer ce qui relève bien de l'assistance terminologique et linguistique, et fixer certaines limites relatives à la nature de ce service, tout en s'efforçant de mesurer son efficacité.

Pour certaines personnes qui demandent une consultation, la frontière n'est pas nette entre ce qui est terminologique et ce qui est traductionnel, entre les conseils stylistiques ou phraséologiques et la révision de texte, entre l'orthotypographie et la mise en page voire l'édition, entre les données linguistiques ou toponymiques, par exemple, et des données encyclopédiques, historiques ou simplement documentaires. Pour les terminologues non plus d'ailleurs, il n'est pas toujours facile de déterminer jusqu'où peut et doit aller l'assistance, et où commence l'extra-linguistique et... l'abusif. De même, ceux-ci sont en droit d'obtenir de leurs usagers tous les renseignements de base nécessaires à la conduite de la recherche et indispensables pour qu'il y ait bel et bien adéquation entre le besoin et le service, faute de quoi il ne saurait y avoir de satisfaction ni d'un côté ni de l'autre.

Mais justement, comment peut-on évaluer cette satisfaction?

Par des sondages ou des enquêtes? C'est possible et cela s'est déjà fait. Mais la demande est aussi là pour rappeler l'utilité du service, conforme à la mission de correction et d'enrichissement de la langue confiée à l'organisme. Les usagers deviennent des habitués dont les besoins se raffinent. Les lémoignages d'appréciation écrits et verbaux sont nombreux. Tel slogan publicitaire reflète bien l'avis linguistique donné. Tel journaliste ose employer un néologisme de préférence à un anglicisme répandu. Pas besoin de publicité, le bouche à oreille suffit! Et les résultats des recherches terminologiques ponctuelles contribuent à l'enrichissement de la banque de terminologie du Québec, fréquemment réinterrogée ensuite avec succès à l'occasion de nouvelles consultations, sur des fiches issues de ces recherches antérieures. 
Bien sûr, l'efficacité du service n'est pas sans faille: l'accès par téléphone exige de la patience (la télécopie lui vient maintenant à la rescousse), les meilleurs conseils ne sont pas toujours suivis, pour quantité de raisons qui vont de l'impossibilité pour le demandeur de modifier suffisamment un texte, à la difficulté d'être sur la même longueur d'onde linguistique, en passant par la déception de l'usager qui n'entend pas la confirmation qu'il aurait souhaitée...

Un service d'assistance terminologique et linguistique constitue à n'en pas douter un excellent miroir de l'intérêt du public pour les questions de langue et il se trouve au confluent des besoins terminologiques et linguistiques, prouvant que la qualité de la langue exige une parfaite imbrication des différentes disciplines linguistiques et, pour les terminologues, l'orchestration d'une vaste gamme de ressources.

\section{BIBLIOGRAPHIE}

CÉLESTIN, Tina, GODBOUT, Gilles et Pierrette VACHON-L'HEUREUX (1984): Méthodologie de la recherche terminologique ponctuelle: Essai de définition, Québec, Office de la langue française.

OFFICE DE LA LANGUE FRANÇAISE (1984): Énoncé d'une politique relative à l'emprunt de formes linguistiques étrangères.

OFFICE DE LA LANGUE FRANÇAISE (1985): Enoncé d'une politique linguistique relative aux québécismes. 